\title{
Atmospheric Radiation Monitor
}

\author{
M.A. Leigui de Oliveira, C.J. Todero Peixoto, M.S.A.B. Leão, V.P. Luzio* \\ Centro de Ciências Naturais e Humanas (CCNH) \\ Universidade Federal do ABC (UFABC) \\ Rua Santa Adélia, 166, Santo André, SP, Brazil \\ E-mail: vitorprestes@yahoo.com.br
}

\author{
A.F. Barbosa, H.P. Lima Jr, A.B. Vilar, R.G. Gama, V.A. Ferraz \\ Centro Brasileiro de Pesquisas Físicas (CBPF) \\ Rua Xavier Sigaud, 150, Rio de Janeiro, RJ, Brazil
}

\begin{abstract}
The Atmospheric Radiation Monitor (MonRAt) is a compact experiment aiming to measure the fluorescence radiation in the atmosphere initiated by cosmic rays with energies between $100 \mathrm{PeV}$ and $100 \mathrm{EeV}$. It is composite by a mirror focusing on a multianodic photomultiplier with an ultraviolet filter and the data acquisition boards. The monitor is intended to contribute in problems such as the study of fluorescence radiation yield in the atmosphere parameterized with atmospheric conditions.
\end{abstract}

Keywords: Cosmic rays, Extensive air showers, Atmospheric fluorescence.

4th School on Cosmic Rays and Astrophysics,

August 25-September 04, 2010

Sao Paulo Brazil

${ }^{*}$ Speaker. 


\section{Introduction}

Primaries from cosmic radiation colide in the upper atmosphere with atomic nuclei generating particles which can travel and colide with other nuclei. The number of particles increases, reaches a maximum, then decreases when the particles start to lose their energy through ionization and excitation of air atoms and molecules. The cascade of particles - called Extensive Air Shower (EAS) - travels to Earth's surface and in its path, nitrogen molecules are excited, mainly by the charged particles of the EAS electromagnetic component, i.e. electrons and positrons. During the deexcitation of nitrogen molecules, fluorescence light is generated which is isotropically emitted with a wavelength spectrum between 300 and $450 \mathrm{~nm}$. In the end of 60's, the detection of EAS using nitrogen fluorescence light has been demonstrated to be successful [1] and nowadays is widely used by several important experiments [2], [3], [4], [5], [6]. The atmosphere is used as a giant scintillator which gives informations about the the EAS longitudinal development. Then, one can estimate the energy and the composition of the primaries. The ammount of fluorescence light created by a huge number of electrons is intense enough to be detected even by photomultipliers placed some kilometers away from the emission point.

To perform the EAS measurements, the fluorescence yield must be parameterized with air composition, density, pressure and temperature. Absolute fluorescence yield is known with uncertainty around $1 \%$ for some electron energies and some air pressures and temperatures [7], [8], [9], [10], [11], [12]. The extrapolation of these results are in present the main source of systematic errors in the determination of EAS parameters by fluorescence telescopes [13]. The fluorescence yield is proportional to charged particles energy deposit in air and varies from 3.0 to 5.6 fotons $/ \mathrm{m}$ per charged particle as a function of altitude. Thus, for a given shower development depth (altitude $h$ within $\Delta x$ ), one can calculate the number of fluorescence photons at the shower axis by:

$$
N_{\gamma}(h)=Y(\lambda, p, T) \cdot \Delta x
$$

where $Y(\lambda, p, T)$ is fluorescence yield which depends on the wavelength, pressure and temperature and $\Delta x$ is the transversed path.

\section{The MonRAt concept}

The Atmospheric Radiation Monitor [14] (MonRAt ${ }^{1}$ ) is a compact telescope designed to detect fluorescence photons generated in the atmosphere by ultra-high energy cosmic rays showers. The detection principle of MonRAt is shown in Fig.1. At the bottom of this figure, the telescope design in Solid Works [15] and a photography of the already assembled mecanical structure are also shown. A 64 pixels MultiAnodic PhotoMultiplier Tube (MAPMT) - Hamamatsu H7546B — will be placed at the focus of a parabolic mirror mounted in a newtonian telescope setup. In front of the MAPMT photocathode, filters will be positioned to select light with wavelength in the near ultraviolet region $(300 \mathrm{~nm}<\lambda<450 \mathrm{~nm})$.

\footnotetext{
${ }^{1}$ From the Portuguese, Monitor de Radiação Atmosférica.
} 

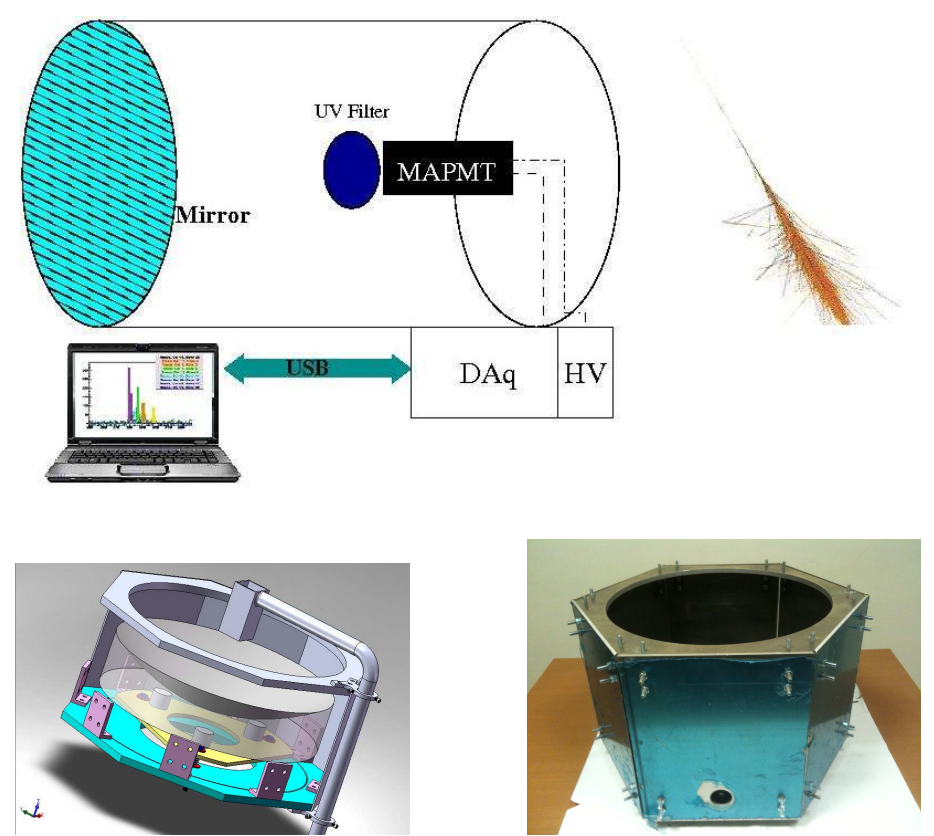

Figure 1: Top: the MonRAt concept. Bottom-left: Solid Works model of its parts; bottom-right: a photography of the assembled strucuture.

\subsection{Simulations}

The mirror geometry limits the optical aberrations and must be produced such that the spot at the focus cannot be bigger in area than one MAPMT pixel, i.e. $2 \times 2 \mathrm{~mm}^{2}$. Therefore, a detailed raytracing software has been written in order to simulate the photons propagations and to choose the best geometrical parameters for the telescope. All dimensions, of the mirror and of the MAPMT, have been implemented and shadow effects considered. The pixel upon which the photons have impinge can be selected for analysis.

Several simulations were done with mirrors that follow the equation of the elliptic paraboloid: $a^{2} z=\left(x^{2}+y^{2}\right)$. The diameter of the mirror was set at $300 \mathrm{~mm}$ and position of the MAPMT at $100 \mathrm{~mm}$ on the axis of the mirror. We varied then the concavity factor $a$, looking for value that would produce the desired image size, without imposing too many technical difficulties for the manufacture of the mirror. The following figures present some results for $a=20 \mathrm{~mm}^{1 / 2}$. Accordingly, a track in the atmosphere that focuses on the diagonal of the pixels is observed under zenith angles in the range $-7.1^{\circ}<\theta<7.1^{\circ}$, giving a good coverage for this track.

In Fig. 2 are presented the histograms of the arrival positions of the simulated photons on the photocathode. The histograms were done with $50 \times 50$ bins $^{2}$, therefore, with much higher resolution than required. Note that in all cases the image formed is smaller than a MAPMT pixel. Note also, for the figure (b) that the structure of the aberration (coma, in this case) is revealed by the simulations.

In order to estimate the amount of light to be collected by MonRAt we simulated a library of showers using CORSIKA [16] — version 6.5021 with SIBYLL 2.1 [17]. For seven fixed energies $\left(10^{17}, 10^{17.5}, 10^{18}, 10^{18.5}, 10^{19}, 10^{19.5}\right.$ and $\left.10^{20} \mathrm{eV}\right)$ we simulated 1000 showers initiated by protons. 
(a)

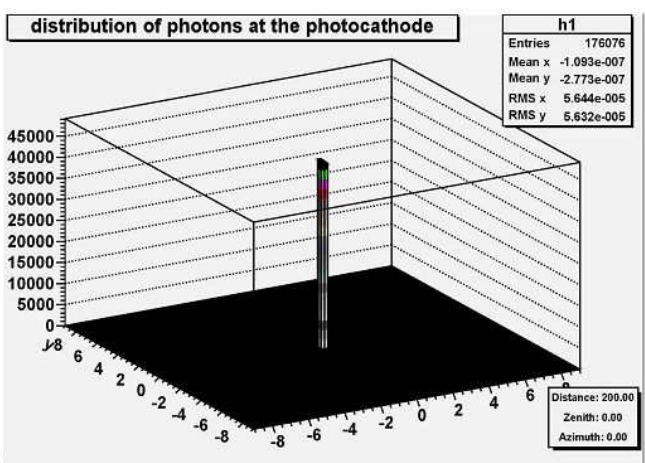

(c)

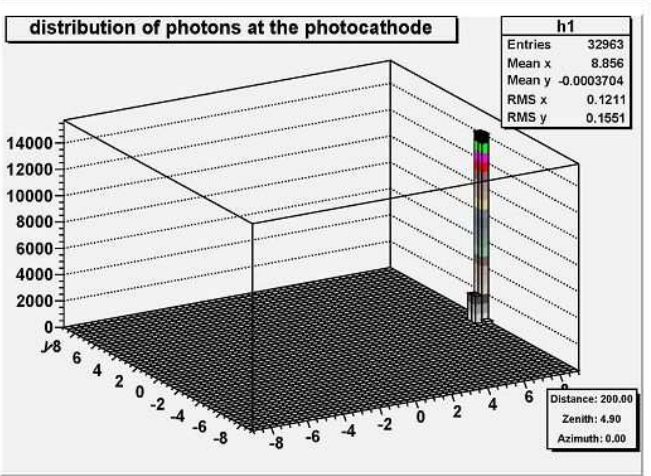

(b)

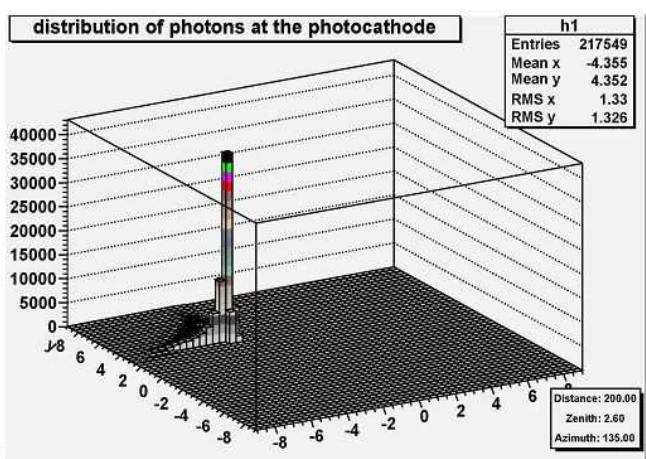

(d)

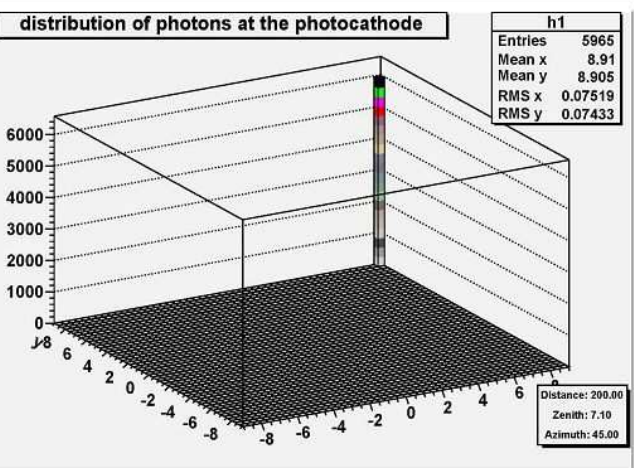

Figure 2: Distributions of positions in the photocathode for photons with incidence: (a) zenithal: $(\theta, \phi)=$ $\left(0^{\circ}, 0^{\circ}\right)$; (b) $(\theta, \phi)=\left(2.6^{\circ}, 135^{\circ}\right)$; (c) $(\theta, \phi)=\left(4.9^{\circ}, 0^{\circ}\right)$; and (d) $(\theta, \phi)=\left(7.1^{\circ}, 45^{\circ}\right)$. The distances listed in the lower boxes are between the photocathode and the shutter.

The particles are observed from the top of the atmosphere until the sea level with longitudinal development in steps of $5 \mathrm{~g} / \mathrm{cm}^{2}$. The zenith angles were sorted between 0 and $60^{\circ}$ and we applied a thinning factor of $10^{-5}$.

The number and the energy of electrons and positrons have been read for each shower at each longitudinal level. Taking into account standard parameterizations of the atmosphere it is possible to estimate the air density, pressure and temperature at each level. Thus, for each particle, a BetheBloch equation is used to calculate the deposited energy due to ionization processes at the given particle energy. Finally, the fluorescence photons yield is calculated through parameterizations formulas chosen among [9] and [10]. A detailed study on the possible combinations of these formulas and their influence on the production of photons at the shower axis is described elsewhere in this school [18].

As the photons are emitted isotropically, their fraction directed towards the telescope is given by the ratio between the mirror solid angle to the total $\left(\Omega_{\text {mirr }} / 4 \pi\right)$. The fluorescence photons that travel to the telescope are attenuated applying molecular and aerosols transmission coefficients $\left(T^{m}\right.$ and $T^{a}$ ). Therefore, multiplying in each level the photon yield at the shower axis by the transversed thickness, by the transmition coefficients and by the solid angle fraction, we can find the number of photons impinging the telescope:

$$
N_{\gamma}^{t e l}=Y \cdot \Delta x \cdot T^{m} \cdot T^{a} \cdot \frac{\Omega_{m i r r}}{4 \pi}
$$


In the following graphics, we show the results for some selected energies. For events with at least one photon impinging on the mirror, the histograms show distributions of important quantities, respectively, from the left to the right:

1. Distribution of shower maximum elevation angles $\left(\alpha_{\max }\right)$ in the telescope field of view. The mean of these distributions indicate the ideal alignment for MonRAt at a given energy;

2. Distribution of shower axis impact parameters $(R p)$. The lowest global $R p$ gives the smallest transit time of photons through the MAPMT, e.g. an EAS with $R p=1 \mathrm{~km}$ will shine the photocathode in $\sim 233 n s$, which gives, suposing a vertical incidence, a time difference of $\sim 29 n s$ between neighboring pixels (the electronics was designed to have time resolution of $5 n s)$;

3. Distribution of number of photons arriving at the telescope.

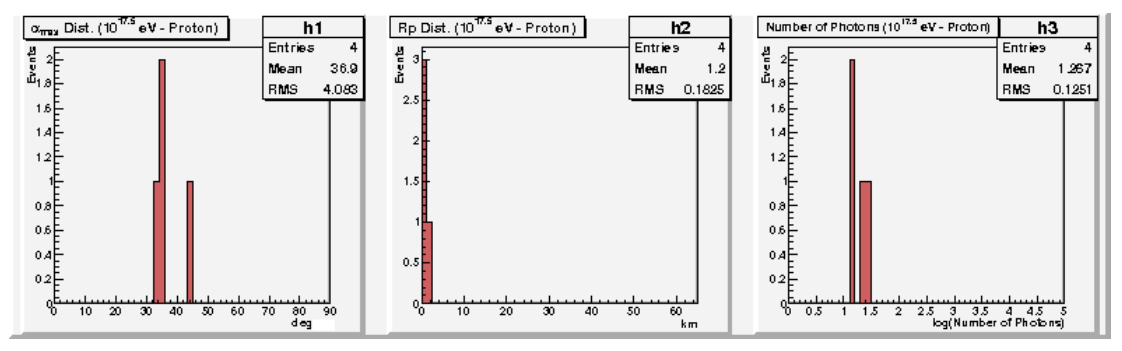

Figure 3: Distributions for $10^{3}$ proton showers of $10^{17.5} \mathrm{eV}$.

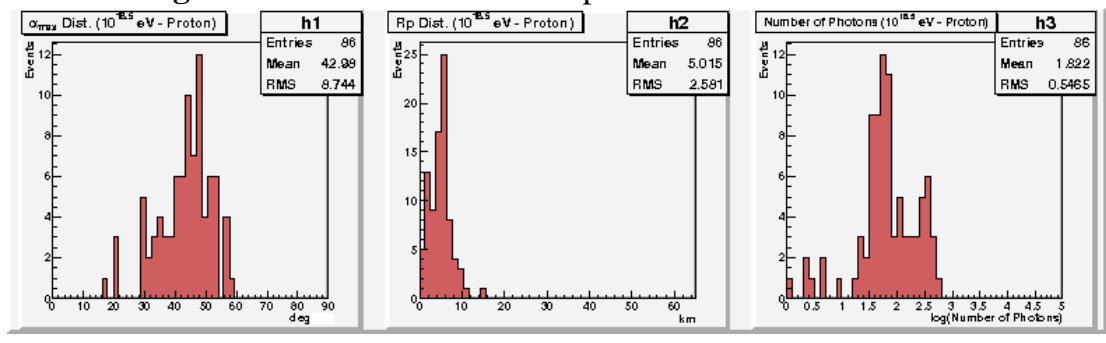

Figure 4: Distributions for $10^{3}$ proton showers of $10^{18.5} \mathrm{eV}$.

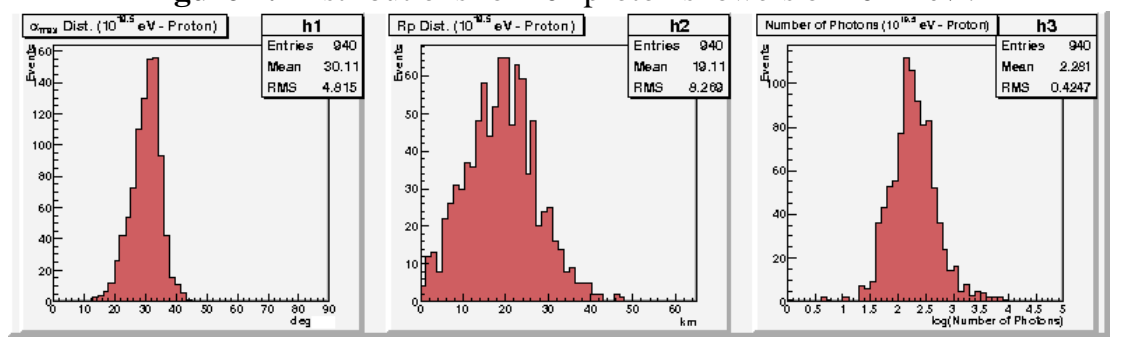

Figure 5: Distributions for $10^{3}$ proton showers of $10^{19.5} \mathrm{eV}$.

These simulations showed that the energy threshold for detecting EAS is $10^{17.5} \mathrm{eV}$ and that the range of the telescope is about $40 \mathrm{~km}$.

The simulations results were also important for designing the acquisition system and for setting the dynamic range of the experiment. For a parabolic mirror with $30 \mathrm{~cm}$ of diameter and concavity 
factor $a=20 \mathrm{~mm}^{1 / 2}$, we obtained $-4,9^{\circ}<\theta<4,9^{\circ}$ for the MAPMT field of view and the solid angle viewed by one pixel is $\Omega_{p i x}=1.44 \times 10^{-4} \mathrm{srad}$. One pixel views a shower with maximum depth $1 \mathrm{~km}$ away developing in $71.2 \mathrm{~ns}$ and $21.35 \mathrm{~m}$. A given vertical proton shower of $10^{18} \mathrm{eV}$ produces $10^{8}$ electrons in the maximum depth. The $\mathrm{S} / \mathrm{N}$ ratio is proportional to the mirror diameter and inversely proportional to $\sqrt{\Omega_{\text {pix }}}$, so we estimate $S / N \sim 7 \times 10^{5}$ per pixel for such shower.

\section{The data acquisition system}

The data acquisition system will consist of sets of pre-amplifiers in the front-end of the MAPMT and FPGA-based boards (called DPMs) able to record trigger times and waveforms from each channel. Each DPM receives and processes the signals from 4 pixels and the data are sent to a computer by USB ports.

The DPMs saturate for signals with amplitude of $1 \mathrm{~V}$, therefore is preferable to observe single photon peak at low amplitudes $(\sim 30 \mathrm{mV})$ such that the dynamical range of DPM can cover as many photons as possible. In Fig.6, we present the single photon spectrum for a blue led flashing at the central pixel with a frequency of $10 \mathrm{kHz}$. The MAPMT high voltage was set to $950 \mathrm{~V}$ and we estimate a gain of $1.5 \times 10^{6}$, in accordance to the manufacturer specifications [19].

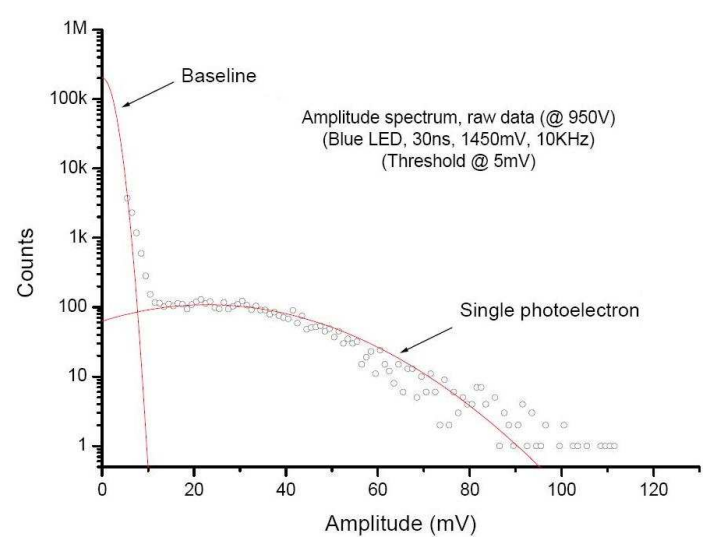

Figure 6: Single photon spectrum.

\subsection{Front-end electronics}

The signals coming from the MAPMT must be pre-amplified, since they have low amplitude and short duration $(\sim 30 n s)$. Thus it is required a circuit with low noise, broadband and performing the functions of the integrator, amplifier and filter. Subsequently, the signal will be taken to the DPMs.

The chosen active element of the pre-amplifier circuit is the operational amplifier OPA657 FET, shown in Figure 7. The first stage works as an integrator-differentiator, producing the signal format. The variable resistor RShape allows fine adjustment of the gain and pre-amplified waveform. The second stage amplifies the signal, multiplying its amplitude by a constant value (within a range of frequencies). The MonRAt detector uses sets of pre-amplifiers as shown in Fig.7, and each set consists of 5 pre-amp circuits. The circuits are powered at $+8 \mathrm{~V}$ and $-8 \mathrm{~V}$. 

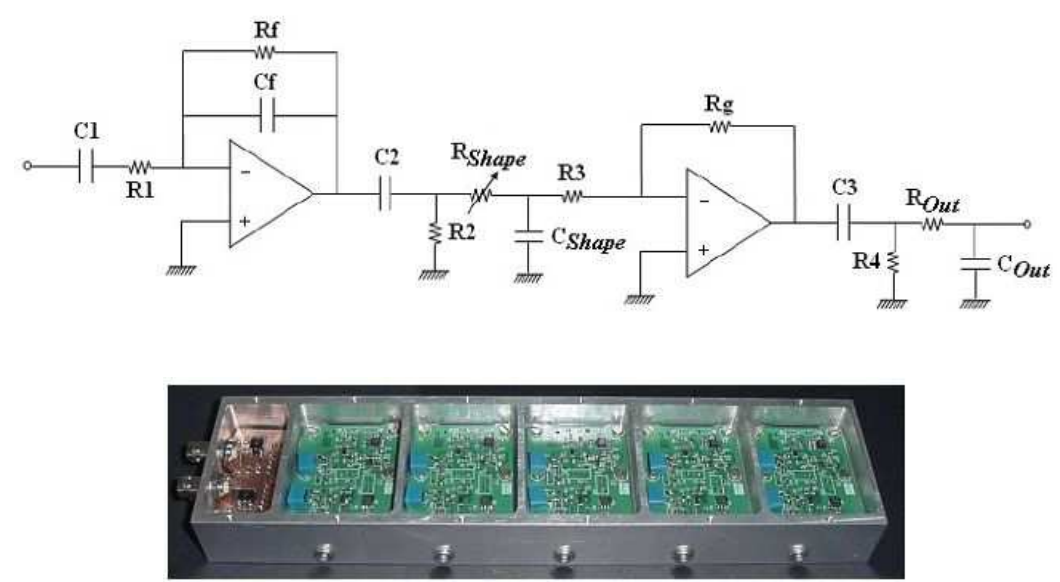

Figure 7: Upper part: pre-amplifier circuit. Lower part: photography of a set of circuits.

\subsection{Data processing module}

The Data Processing Module (DPM) is an electronic circuit developed for data acquisition that has as main processor a programmable logic FPGA device. It is responsible for digital signal processing and communication with the computer via USB port. The module has two analogue to digital converters, 2 channels each, totaling 4 analog input channels with 12 bit resolution. Measurements of signal amplitudes are made at a rate of 60 MSPS for signals with amplitude between $-1 V$ and $+1 V$. The DPM also has a time-digital converter which measures the delay time between the triggered anode signals and a reference given by the instant of trigger of the $12^{\text {th }}$ dynode of the MAPMT. The TDCs measure time intervals with resolution of $5 \mathrm{~ns}$. A prototype of a DPM board and a setup for first the 16 channels are shown in Fig.8.
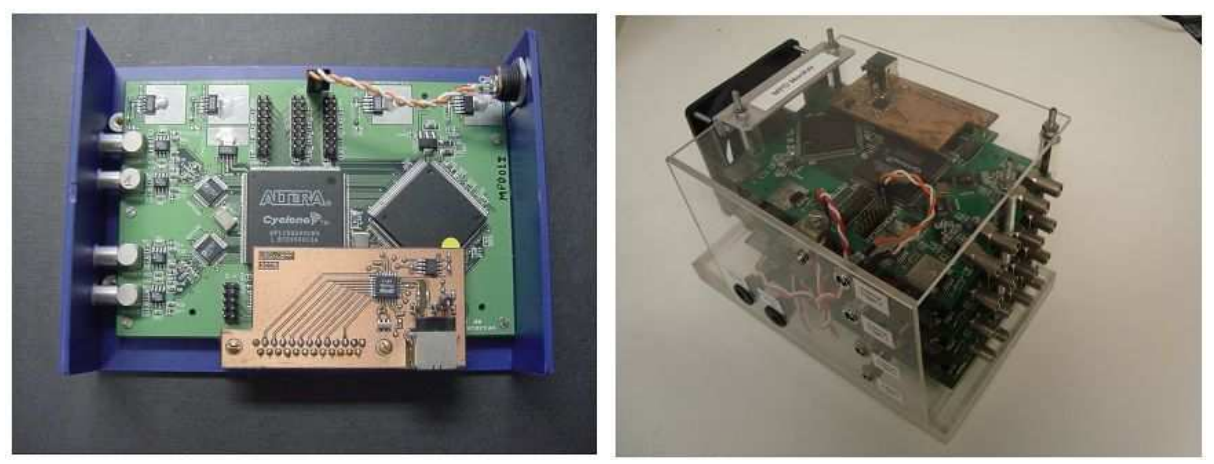

Figure 8: To the left: a photography of 1 DPM board. To the right: the setup for 16 pixels.

For use in MonRAt were grouped four DPMs, taking 16 analog input channels for reading signals from the pixels of the MAPMT. The assembly of the modules can be seen in figure 8 . In addition to the analog inputs, the DPM has a digital input for trigger, which activates a process of acquisition of events from the analog channels in their positive transition. The set of DPMs has a single power input shared by all with supply voltage of $+6 \mathrm{~V}$ and maximum current of $2.8 \mathrm{~A}$. There is also another entry for feeding the cooling fan, with a voltage of $+12 \mathrm{~V}$ and maximum current of $0.15 \mathrm{~A}$. 


\subsection{Calibration of the DPMs}

To obtain the final value of the signal amplitude is necessary to establish a relationship between the digital value converted by the ADC and analog input voltage applied on the DPM. Thus, a measurement system was set up for calibration of the channels of DPMs.

Pulses of known amplitude and width were provided and the converted values stored in file. Since the relationship between pulse amplitude and the converted values is linear, angular and linear coefficients were obtained through a fit to the data. We acquired 3000 measurements for each amplitude value in the range of $-1 \mathrm{~V}$ to $+1 \mathrm{~V}$ with steps of $100 \mathrm{mV}$. The mean and standard deviation were calculated and plotted to obtain the linear fit coefficients. The graph in figure 9 shows the result of an adjustment for the channels of a DPM.

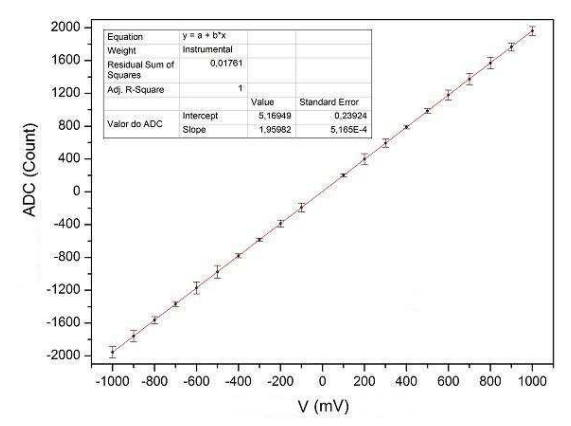

Figure 9: Linear fit to calculate the linear constants (Intercept) and angular (Slope) of a typical DPM channel.

This calibration procedure was repeated for the 16 available DPMs and the values of conversion (ADC-voltage) are implemented as constants in the data acquisition software. Moreover, such a calibration was done on the lab bench. We are currently developing a system (software and hardware) to perform such calibrations before each data taking and in situ. That is, before a night of data taking the calibration procedure will be performed automatically, adjusting the parameters of the conversion for the site where the experiment will work under the same atmospheric conditions, especially temperature.

\section{Software}

The data acquisition program was developed using the platform ROOT [20], version 5.26.2000, and the library of functions for the USB transceiver. The program is written in $\mathrm{C}++$ and is object oriented. The main body of the program consists of 4 classes: the main class responsible for displaying the user interface and managing the actions necessary for the operation of the module, one class which declares the object for each DPM, one class for communication with DPMs and one for communication with the USB ports. The main class has routines for signal processing, such as obtaining the amplitude, the baseline and the charge.

Figure 10 presents a preview of the acquisition program during a run of data taking. The software has options for displaying graphics and writing to files, chosen by the user via checkboxes. The recording options include the types of files that can be recorded (waveforms or tables), the nomenclature or initial path of the files generated and the DPM data source. Data generated in 
these output files will be used to reconstruct the events. Only signals from a single DPM can be viewed at a time, which is selected via the tabs above. Event counters for each device are shown on the screen and a status bar displays terms of connections and messages to the user.

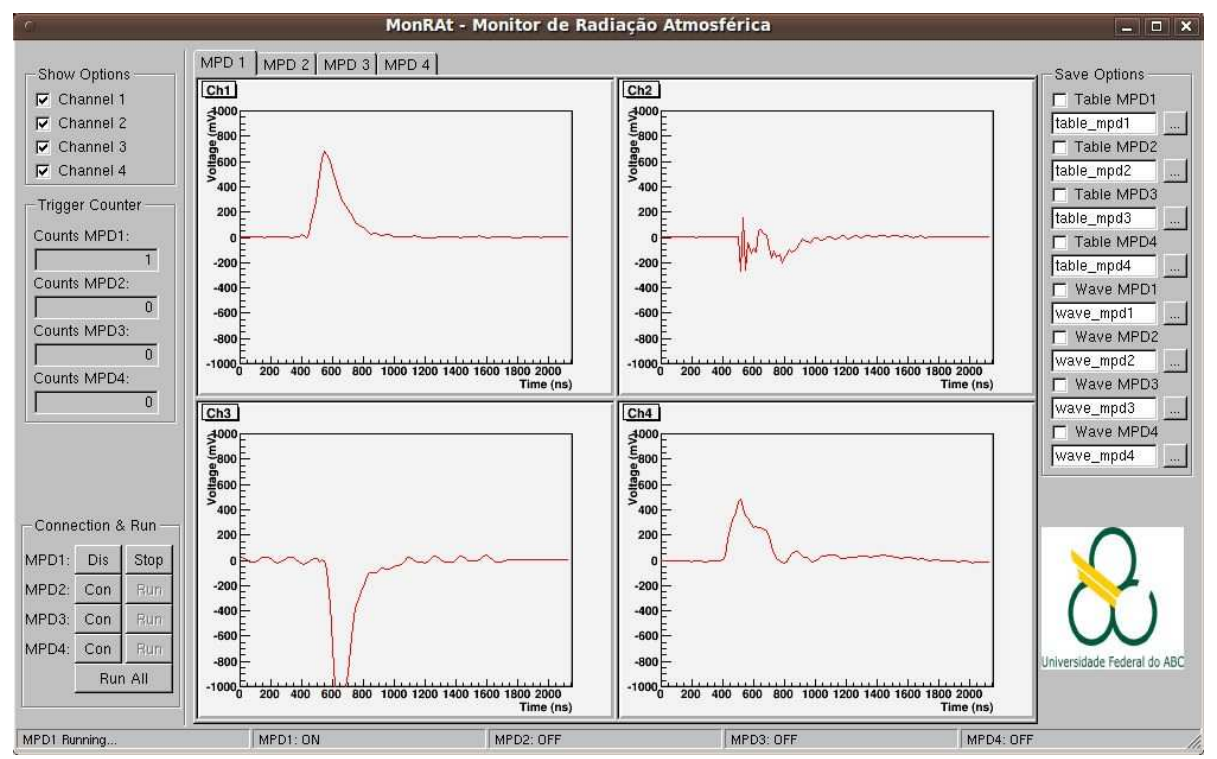

Figure 10: Graphical interface of the data acquisition software.

\section{Conclusions}

We presented the design of the MonRAt detector, its expected performance and the present status of the experiment. MonRAt is designed for the detection of fluorescence radiation generated by EAS particles of primary energies greater than $10^{17} \mathrm{eV}$. Studies about the detector optical properties, details for the electronics and the simulations performed to better understand the MonRAt telescope has been presented. The detector is under construction - the last DPMs are being produced and final assemblies of the mechanical structure being finished - and the full setup will be soon available and we will be able to measure real events, pioneering detecting the fluorescence radiation in Brazil.

\section{Aknowledgements}

The financial support for this work was given by the Brazilian foudations CNPq (Conselho Nacional de Desenvolvimento Científico e Tecnológico) and FAPESP (Fundação de Amparo à Pesquisa do Estado de São Paulo). The authors are thankful to our colleagues at UFABC and CBPF for the facilities used in the development of this work. 


\section{References}

[1] T. Hara et al., Acta Phys. Acad. Sci. Hung., 29, Suppl. 3 (1970) 361.

[2] R. M. Baltrusaitus et al., Nucl. Instr. and Meth. in Phys. Res.: A240 (1985) 410.

[3] T. Abu-Zayyad et al., Nucl. Instr. and Meth. in Phys. Res.: A450 (2000) 253.

[4] J. Abraham et al., Nucl. Instr. and Meth. in Phys. Res.: A523 (2004) 50;

[5] M. Fukushima, Prog. Theo. Phys. Suppl., 151 (2003) 206.

[6] T. Ebisuzaki for the JEM-EUSO Collaboration, Proc. of XVI ISVHECRI, USA (2010) [arXiv:1101.1909].

[7] A. N. Bunner, Cosmic Ray Detection by Atmospheric Fluorescence, PhD Thesis, Cornell Univ. (Feb. 1967).

[8] G. Davidson and R. O’Neil, J. Chem. Phys., 41 (1964) 3946.

[9] K. Kakimoto, et al., Nucl. Instr. and Meth. in Phys. Res.: A372 (1996) 527.

[10] M. Nagano et al., Astropart. Phys., 20 (2003) 293; 22 (2004) 235.

[11] J. W. Beltz et al., Astropart. Phys., 25 (2006) 129.

[12] P. Collin et al., astroph/06121110.

[13] V. de Souza, G. Medina-Tanco and J. A. Ortiz, Astropart. Phys., 25 (2006) 129.

[14] M. A. Leigui de Oliveira et al., Atmospheric Radiation Monitor, Proc. of the $31^{\text {st }}$ ICRC, Łódź (2009), Session HE.2.4, ID=97.

[15] Solid Works, http://www.solidworks.com

[16] D. Heck, J. Knapp, J. N. Capdevielle, G. Schatz, and T. Thouw, Report FZKA 6019 (1998), Forschungzentrum Karlsruhe; http://www-ik.fzk.de/ heck/publications/fzka6019.pdf

[17] R. S. Fletcher et al., Phys. Rev. D, D50 (1994) 5710.

[18] C. J. Todero Peixoto, M. A. Leigui de Oliveira, and M. S. A. B. Leão, Study of Number of photons at axis Shower with different $d E / d x$ and Fluorescence Yield, Proc. of the $4^{\text {th }}$ Sch. on CR and Astroph., Santo André, Brazil (2010).

[19] http://www.hamamatsu.com

[20] http://root.cern.ch/root 